\title{
Identificação preliminar dos serviços ecossistêmicos de provisão prestados pelo manguezal no rio Tubarão e no rio Ceará-Mirim (RN- Brasil)
}

\author{
Preliminary identification of the ecosystem services provided by the mangrove in the \\ Tubarão river and the Ceará-Mirim river (RN-Brasil)
}

GUEDES $^{1}$, D. R. C.; $\operatorname{COSTA}^{2}$, D. F. S.; CESTARO, L. A ${ }^{3}$. dayane.geo10@gmail.com;

\begin{abstract}
Resumo
O manguezal proporciona uma série de Serviços Ecossistêmicos (SE) necessários para a manutenção das comunidades humanas que habitam o seu entorno. Esta pesquisa foi realizada em duas áreas de estudo, com enfoque no ecossistema de manguezal em estuários que apresentam condições físicas diferentes, com o objetivo identificar os possíveis serviços ecossistêmicos de provisão prestados pelo ambiente de manguezal no Rio Tubarão, localizado no litoral setentrional do Rio Grande Norte e no manguezal do rio Ceará-Mirim, situado no litoral oriental potiguar. Para a identificação dos SE, foi utilizada a classificação CICES (Common International Standard for Ecosystem Services). Verificou-se que o manguezal desempenha um papel importante para as comunidades locais, que se beneficiam de forma direta e indireta dos bens e serviços prestados.
\end{abstract}

Palavras-chave: Manguezal; Serviços Ecossistêmicos; CICES

\begin{abstract}
The mangrove provides a series of Ecosystem Services (SE) necessary for the maintenance of the human communities that inhabit its surroundings. This research was carried out in two study areas, focusing on the mangrove ecosystem in estuaries that present different physical conditions, with the objective of identifying the possible ecosystem services provided by the mangrove environment on the Tubarão River, located on the northern coast of Rio Grande North and in the mangrove of the river Ceará-Mirim, located in the eastern seaboard of the Potiguar. For the identification of the SE, the CICES (Common International Standard for Ecosystem Services) classification was used. It has been found that mangroves play an important role for local communities, which benefit directly and indirectly from the goods and services provided.
\end{abstract}

Keywords Mangrove; Ecosystem Service; CICES

\section{INTRODUÇÃO}

O manguezal é um ecossistema de transição entre o ambiente marinho e terrestre, localizado principalmente nos estuários e estando sujeitos ao regime de marés, sendo considerado um dos ecossistemas mais dinâmicos do planeta. Apresentando uma importância que vai além do aspecto ecológico, muitas comunidades humanas que vivem no litoral tiram seu sustento dos manguezais através da pesca artesanal (DAWES et al., 1999; ALONGI, 2002; VANNUCCI, 2002; POLIDORO et al., 2010).

Os atributos físicos do meio influenciam no ecossistema de manguezal, dessa forma as características climáticas têm papel importante para determinar o grau e o nível de desenvolvimento

\footnotetext{
${ }^{I}$ Dayane Raquel da Cruz Guedes, Departamento de Geografia/Laboratório de Biogeografia, Universidade Federal do Rio Grande do Norte, Caicó-RN, Brasil

${ }^{2}$ Diógenes Félix da Silva Costa, Departamento de Geografia/Laboratório de Biogeografia, Universidade Federal do Rio Grande do 
das áreas de manguezal. Em regiões áridas, esse ecossistema exibe estrutura de pequeno porte, entretanto, em clima subúmido apresentam bosques mais desenvolvidos (LUGO, 1980). Duke et al. (2007) e Bunt (1999) acrescentam que a salinidade é um parâmetro de grande importância ecológica e está diretamente relacionado com a distribuição das espécies de mangue, especialmente em ambientes áridos, onde pode haver a acumulação de sais nos solos.

Dessa forma, o nível de desenvolvimento do manguezal e a salinidade estão vinculados com os diferentes tipos de estuários, sendo mais marcante entre os classificados como negativos/invertidos e positivos. No ambiente de estuário negativos, Miranda et al. (2002) descrevem que são tipicamente encontrados em regiões áridas que apresentam precipitação anual baixa, tendo pouca vazão de água doce nesses sistemas, podendo ser considerados hipersalinos, onde as concentrações de sal excedem algumas vezes $50 \mathrm{gL}-1$. Em estuários positivos, estes valores extremos variam de 0 a 30 gL-1 com relação a distância e proximidade com o oceano. (DEBENAY et al., 1989; SAVENIJE; PAGÈS, 1992; SIMIER et al., 2004).

Nesta perspectiva, no Rio Grande do Norte ocorrem áreas de abrangência dos dois tipos de estuários. No litoral setentrional os ambientes estuarinos demonstram características hipersalinas e no litoral oriental apresentam características de estuário positivo (SILVA et., al., 2005; DIAS et al., 2007; COSTA et al, 2014).

A pesquisa é baseada em duas áreas de manguezais que estão localizados em estuários que apresentam condições físicas diferentes. Um dos ambientes estuarinos estudado, possui aporte de água doce proveniente do lençol freático das dunas adjacentes e das chuvas, que é o caso do Rio Tubarão (litoral setentrional/semiárido), na Reserva de Desenvolvimento Sustentável Estadual Ponta do Tubarão (DIAS, 2006), caracterizado por ser um ambiente hipersalino. Já no litoral oriental, por apresentar clima subúmido e aporte de água doce permanente, o estuário é caracterizado como positivo, onde nessa área do Rio Ceará-Mirim tem pluviosidade média anual acima de $1.400 \mathrm{~mm}$ (SOARES, 2010).

Os estuários são capazes de sustentar uma intensa atividade biológica, onde podem se fixar várias espécies de animais e vegetais. Isto afirma a importância deste ambiente na economia de muitas comunidades costeiras. (FALCÃO, 2005). Ainda segundo Falcão (2005), a diversidade de espécies que são exploradas pelas comunidades tradicionais, serve de base para subsistência, vindo a beneficiar diretamente as comunidades de pescadores. $\mathrm{O}$ manguezal proporciona uma série de serviços ecossistêmicos necessários para a manutenção das comunidades humanas que habitam o entorno desses ambientes. Considerado como um dos mais produtivos do planeta, este ecossistema propicia alguns serviços como o fornecimento de recursos vegetais e animais para populações humanas (COSTA et al, 2014, POLIDORO et al, 2010). 
A partir dos aspectos físicos que diferem as duas áreas de estudo, esta pesquisa, objetivouse identificar os possíveis serviços ecossistêmicos de provisão prestados pelo ambiente de manguezal no Rio Tubarão e no Rio Ceará-Mirim, classificando-os de acordo com tabela CICES (HAINES-YOUNG E POTSCHIN, 2013).

\section{METODOLOGIA}

2.1 - Área de estudo

Para a pesquisa foram selecionadas duas áreas de manguezal localizadas um no litoral setentrional e no litoral oriental no Estado do Rio Grande do Norte. A primeira área é o manguezal do Rio Tubarão, que se localiza no litoral setentrional e situa-se entre os municípios de Macau e Guamaré - RN. A segunda área de estudo é o manguezal do Rio Ceará-Mirim, que está localizado no litoral oriental potiguar, próximo ao distrito de Estivas até a sua desembocadura, na comunidade Barra do Rio, no município de Extremoz (FIGURA 01).

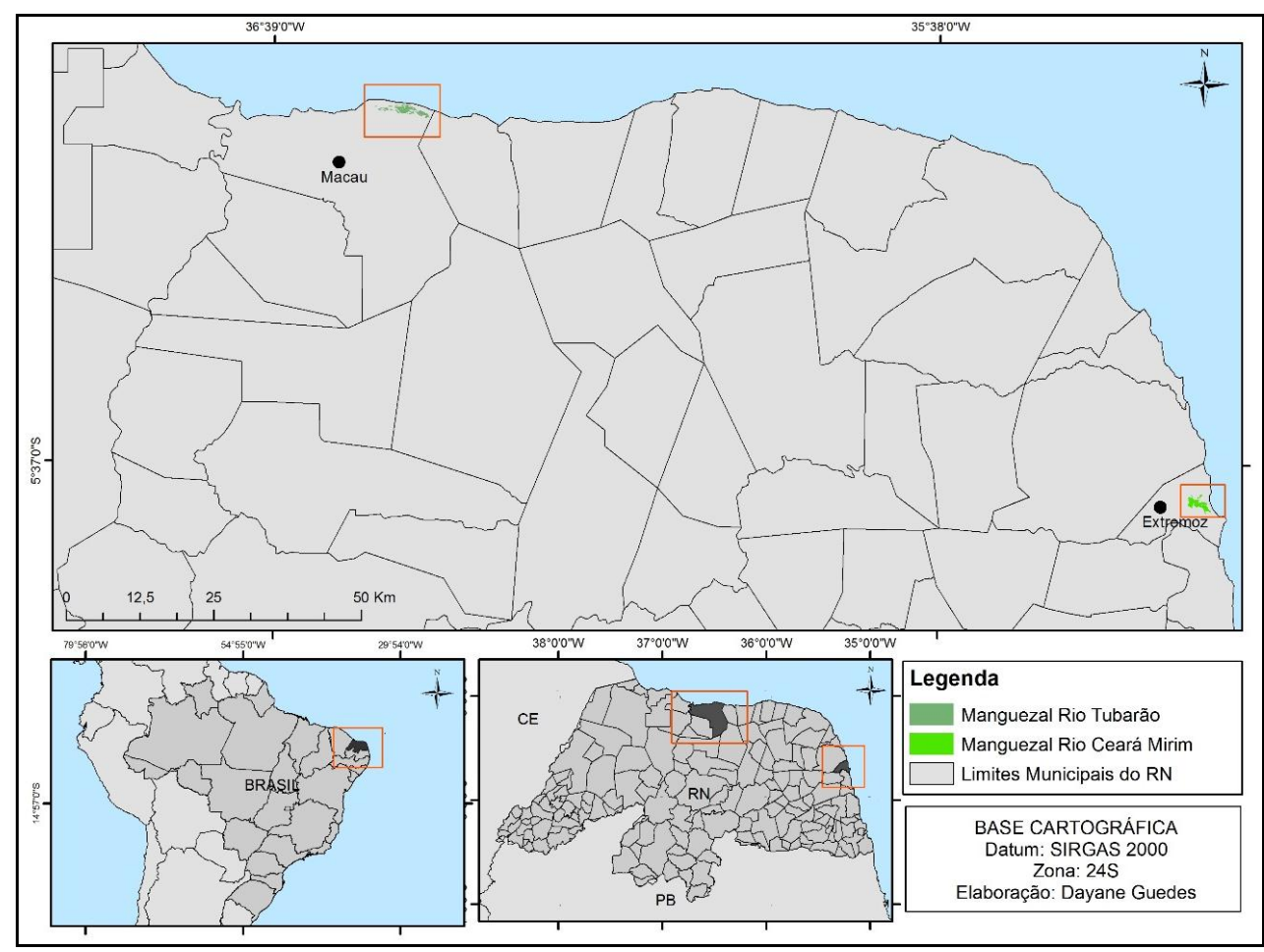

Figura 01 - Mapa de localização do rio Tubarão e do Rio Ceará-Mirim. Fonte - Dados do IBGE. 
2.2 - Procedimentos metodológicos

Para alcançar o objetivo proposto, foi utilizado como conceito chave o aporte teórico elaborado pelo sistema de classificação CICES (Common International Standard for Ecosystem Services) (HAINES-YOUNG E POTSCHIN, 2013). O estudo foi realizado através de levantamentos de dados de campo e cruzamento de informações científicas já publicadas sobre as áreas de estudos.

No artigo foi trabalhado os serviços de provisão que incluem os produtos e materiais obtidos a partir do ecossistema. Isso se deve ao fato de ser o serviço que apresenta importância no contexto social e econômico das comunidades locais. Como a tabela da CICES foi desenvolvida para identificar os serviços de todos os ecossistemas, dessa forma as tabelas são adaptadas aos serviços que são fornecidos pelo ecossistema manguezal.

A tabela CICES é organizada de forma hierarquizada onde trabalha em diferentes escalas, partindo do mais geral para o mais específico (FIGURA 02). A seção, é o nível mais alto e o mais genérico desta estrutura, compreendem três grupos distintos: serviços de provisão, serviços de regulação e manutenção, e serviços culturais. Desta forma, o CICES segue uma estrutura hierárquica como forma de permitir aos usuários selecionar o nível mais adequado para a aplicação da pesquisa (SOUZA et al., 2016).

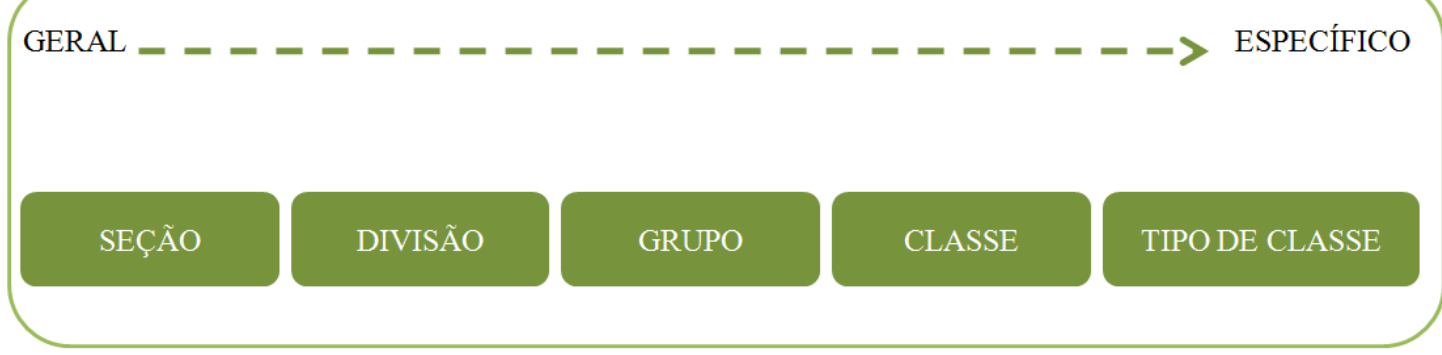

Figura 02 - Estrutura hierárquica da classificação CICES. Fonte - Adaptado de Rabelo (2014).

Foi realizada a elaboração da listagem dos possíveis serviços ecossistêmicos de provisão associados ao manguezal, consultando literatura produzidos para a área de estudo e realizando observações locais. Análise do material bibliográfico, partiu de pesquisas científicas específica da área de estudo, como Dias et al. (2007); Mattos et al. (2012); Rocha Júnior (2011); Queiroz et al. (2014); Lima (2005); Costa (2005) e a visitas de campo ocorreu no período de 28-29 de abril de 2016 e 23 de fevereiro de 2017. 
Vale ressaltar, que os resultados fazem parte da primeira etapa da pesquisa de dissertação, e que contribui para aprofundar o conhecimento acerca das áreas de estudos sobre os produtos fornecido pelo sistema biótico e abiótico pelo ecossistema à população.

\section{RESULTADOS E DISCUSSÕES}

A partir da revisão bibliográfica foram levantados os serviços ecossistêmicos dos manguezais presentes nas áreas de estudos. Os quadros a seguir apresentam os resultados iniciais desta etapa da pesquisa, divididas nas categorias de serviços ecossistêmicos. Vale salientar que os quadros foram modificados, pois nem todos os serviços, proposto pela CICES se aplicam nas áreas de estudo, nessa primeira etapa de pesquisa.

Os serviços de provisão do rio Tubarão estão elencados no quadro 01, abrangem duas categorias de divisão (a nutrição e materiais), distribuídos em três classes (plantas selvagens, algas; animais selvagens e fibras/materiais de plantas e animais para uso direto ou transformação).

Quadro 01 - Potencias serviços ecossistêmicos de provisão do manguezal da RDSEPT. Fonte - Quadro adaptado de Haines-Young e Potschin, 2013.

\begin{tabular}{|c|c|c|c|c|}
\hline SESSÃO & DIVISÃO & GRUPO & CLASSE & $\begin{array}{l}\text { TIPOS DE } \\
\text { CLASSE }\end{array}$ \\
\hline \multirow{3}{*}{ 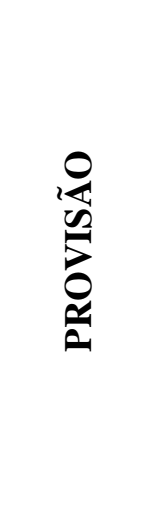 } & \multirow[b]{2}{*}{ NUTRIÇÃO } & \multirow[b]{2}{*}{ Biomassa } & Plantas selvagens, algas & Folha para o gado \\
\hline & & & Os animais selvagens & $\begin{array}{l}\text { Caranguejo-uça, } \\
\text { camarão, siris, } \\
\text { ostras, cavalo- } \\
\text { marinho, peixe } \\
\text { agulha, moluscos }\end{array}$ \\
\hline & MATERIAIS & Biomassa & $\begin{array}{l}\text { Fibras/materiais de } \\
\text { plantas e animais para } \\
\text { uso direto ou } \\
\text { transformação }\end{array}$ & Madeira \\
\hline
\end{tabular}

Na classe de plantas selvagens e algas, Dias et al. (2007) afirmam que as folha das espécies de mangue são utilizadas pelos moradores das comunidades como alimento para o gado caprino e equino. Algumas espécies vegetais possuem glândulas secretoras de sal como forma de adaptação para eliminar o excesso de sal que fica presentes em suas folhas (TOMLINSON, 1986; YE et al., 2005). O sal vai desempenhar um papel importante na vida dos animais, sendo que o sal encontrado nas folhas de mangue se torna suficiente para as necessidades fisiológicas do animal.

Para as comunidades que vivem próximas aos manguezais, os moluscos representam um dos grupos de maior relevância econômica. Nessas áreas, a coleta desses animais pode se constituir 
na principal fonte de renda das famílias envolvidas ou como complemento de outras atividades extrativistas. A pesca é a atividade mais importante na Reserva que incluem pescadores, catadores de caranguejo e marisqueiras (DIAS et al., 2007) (FIGURA 03).

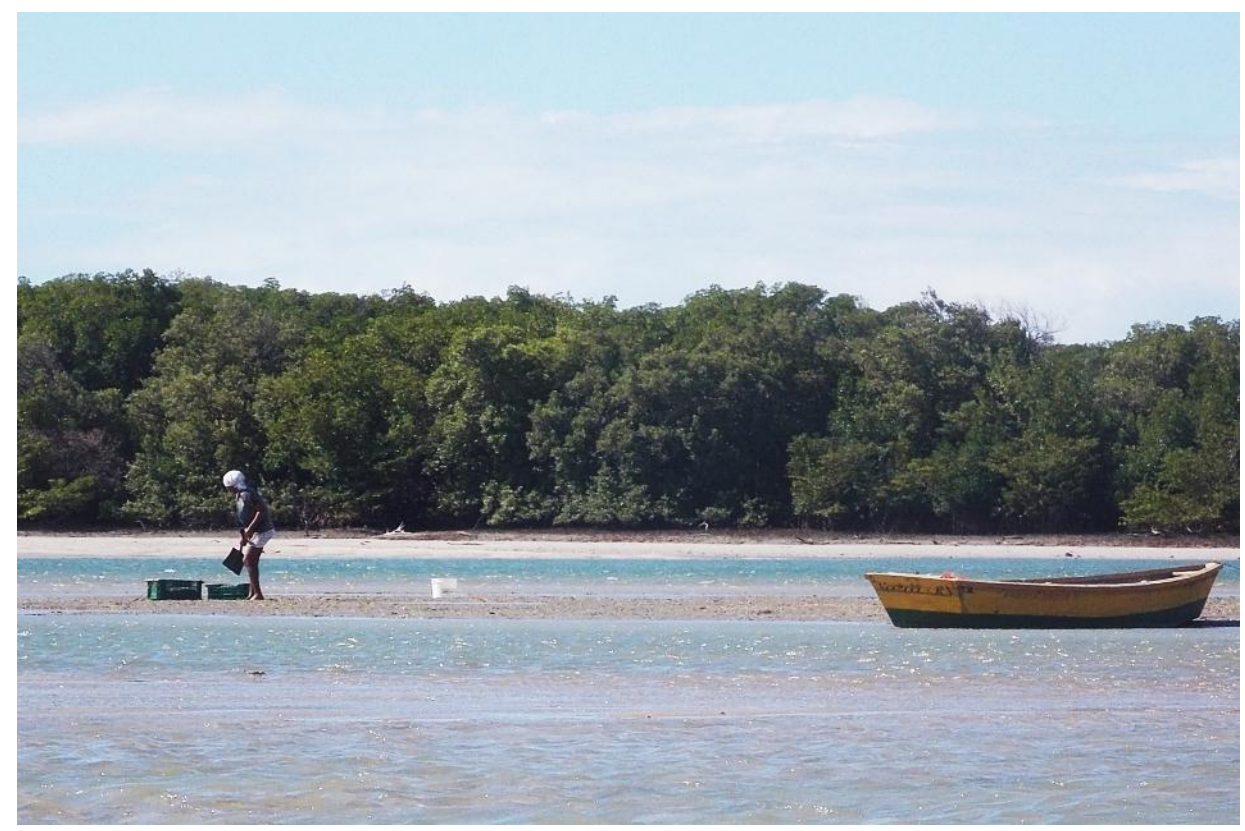

Figura 03 - Marisqueiras catando os moluscos na RDSEPT. Fonte - Guedes, 2016.

Já com relação a fibras/materiais de plantas e animais para uso direto ou transformação utilizadas na RDSETP, a madeira, principalmente de Rhizophora mangle L. (mangue vermelho) é utilizada na construção de casas de taipa, ranchos de pescadores, embarcações, como também lenha para fogões, cercas e embarcações. Mattos et al. (2012) afirmam que as marisqueiras utilizam a madeira da R. mangle para ferver os mariscos, por ser uma forma economicamente viável. Dentre as espécies de mangue, o gênero Rhizophora fornece o melhor carvão de lenha com alto poder calorífico, queima lenta e sem fumaça, afirmando os motivos pelos quais é utilizado pelas marisqueiras (BANDARANAYKE, 1998).

Por sua vez, os serviços de provisão fornecidos pelo manguezal do rio Ceará-Mirim se distribuem de forma diferenciada em comparação à RDSEPT, pois muito produtos que o ecossistema pode fornecer, não são aproveitados pela população local de forma sustentável.

Os serviços englobam três categorias de divisão (a nutrição, materiais e energia) distribuídos em quatro classes: animais selvagens; fibras/materiais de plantas e animais para uso direto ou transformação; materiais genéticos da biota e recurso a base de plantas (Quadro 02). 
Quadro 02 - Potencias serviços ecossistêmicos de provisão no manguezal do rio Ceará-Mirim. Fonte Quadro adaptado de Haines-Young e Potschin, 2013

\begin{tabular}{|c|c|c|c|c|}
\hline SEÇÃ̃O & DIVISÃO & GRUPO & CLASSE & $\begin{array}{l}\text { TIPO DE } \\
\text { CLASSE }\end{array}$ \\
\hline \multirow{4}{*}{ 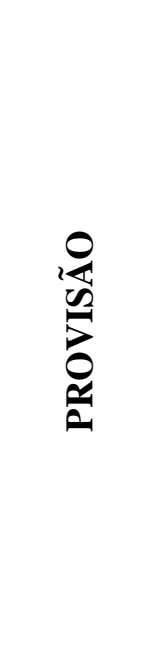 } & NUTRIÇÃO & Biomassa & $\begin{array}{l}\text { Os animais } \\
\text { selvagens }\end{array}$ & $\begin{array}{l}\text { Pesca comercial } \\
\text { /subsistência }\end{array}$ \\
\hline & \multirow[t]{2}{*}{ MATERIAIS } & \multirow[t]{2}{*}{ Biomassa } & $\begin{array}{c}\text { Fibras/materiais } \\
\text { de plantas e } \\
\text { animais para } \\
\text { uso direto ou } \\
\text { transformação } \\
\end{array}$ & Madeira \\
\hline & & & $\begin{array}{c}\text { Materiais } \\
\text { Genéticos da biota }\end{array}$ & $\begin{array}{l}\text { Propágulo para } \\
\text { recuperação de área } \\
\text { de manguezal }\end{array}$ \\
\hline & ENERGIA & $\begin{array}{c}\text { Fontes de } \\
\text { energia à } \\
\text { base de } \\
\text { biomassa }\end{array}$ & $\begin{array}{c}\text { Recursos à } \\
\text { base de plantas }\end{array}$ & Carvão \\
\hline
\end{tabular}

Com relação a presença de animais selvagens, Lima (2005) afirma que a área apresenta a extração de caranguejo, mariscos e pescados ambientes. São para consumo pelas populações que se encontram na área de influência direta do mangue, assim como para comercialização.

Na subdivisão de materiais, referente ao grupo da biomassa, a produção de fibras e outros materiais de plantas, algas e animais para uso direto ou transformação, é caracterizado no manguezal do rio Ceará-Mirim, como a utilização da madeira do mangue, principalmente a espécie da Lagucularia racemosa L.C.F. Gaertn para a produção de varas, que são utilizadas pelos balseiros para remar na travessia do rio (FIGURA 04). E também são utilizadas para as construções de casas de taipa e cercas (COSTA, 2005).
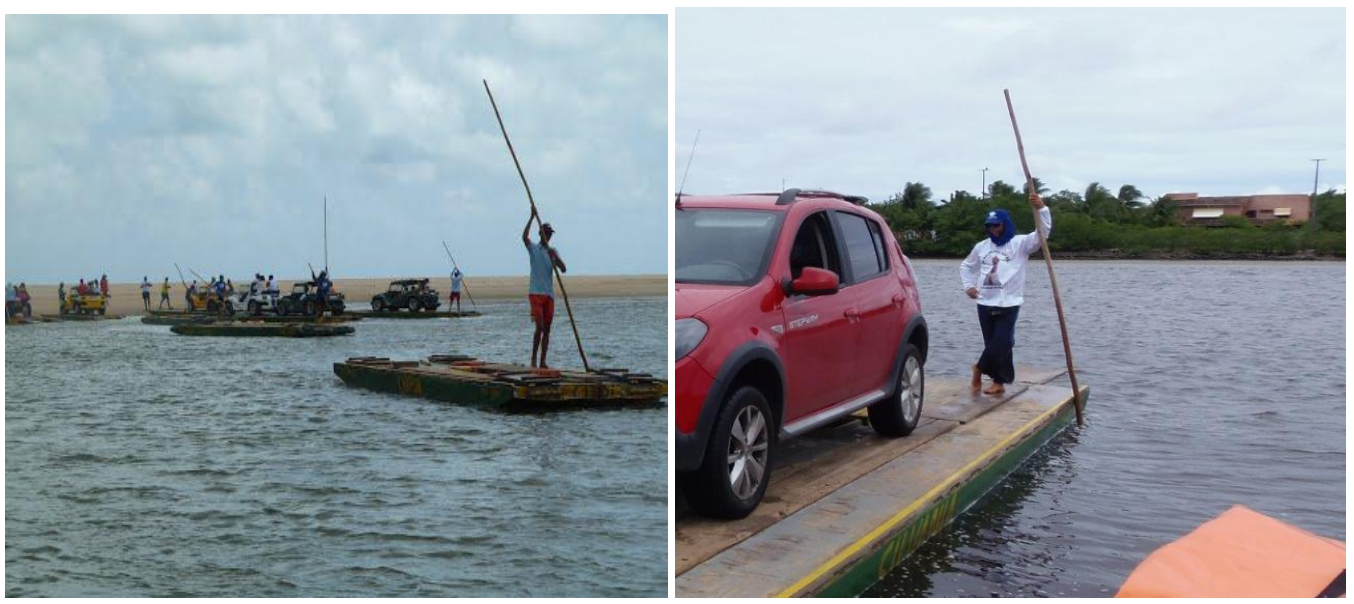

Figura 04 - Balseiros com as lanças retirado da vegetação de mangue. Fonte - Sales, 2014 
Com relação aos materiais genéticos, pode-se enfatizar a utilização dos propágulos produzidos tanto pela R. mangue quanto pelas espécies de Avicennia spp., para ações de recuperação das áreas degradadas no ecossistema. No grupo das fontes de energia a base de biomassa, na classe recursos à base de plantas, destaca-se o uso da madeira para a produção do carvão vegetal que são utilizados como fonte de energia, pelas comunidades locais (COSTA, 2005; LIMA, 2005).

Observa-se que ambas as áreas apresentam SEs semelhantes, no entanto o uso desse ecossistema é o que diferencia, pois, o manguezal do Rio Tubarão está numa área de Unidade de Conservação. Dessa forma, as comunidades locais são consideradas importantes áreas de produção pesqueira do município de Macau, onde cerca de 30\% dos moradores da reserva são pescadores e marisqueiras (IDEMA, 2008). Já no Rio Ceará-Mirim, as pressões antrópicas como as empresas de carcinicultura vem a prejudicar os serviços que o ecossistema vem a oferecer, já que ocupam áreas de manguezais para a instalação dos tanques. Além que, a comunidade local, tem sua economia voltada pela atividade de exploração turística, tendo a pesca com pouca representatividade, mas que fornece sustento para pescadores locais.

\section{CONSIDERAÇÕES FINAIS}

A partir da bibliografia é possível observar que o ecossistema de manguezal desempenhada um papel importante para as comunidades locais que se beneficiam de forma direta e indireta dos bens e serviços prestados pelo manguezal, retirando localmente o sustento de suas respectivas famílias (serviços de provisão), beneficiando-se dos processos naturais que regulam as condições ambientais e atribuindo valor cultural ao ecossistema, conforme evidenciado nos trabalhos científicos consultados.

Vale destacar que os autores utilizados como referência durante a pesquisa não discutem estes benefícios como serviços ecossistêmicos, apenas analisam a existência e importância destes recursos no contexto ambiental e socioeconômico da área de estudo. No entanto, essas pesquisas deram suporte para a identificação e organização dos serviços prestados pelo ecossistema manguezal, em ambas as áreas.

É incontestável a relevância desempenhada pelos manguezais, na qual exercem os mais variados tipos de serviços de provisão, regulação/manutenção e cultural, os quais são fundamentais para a saúde e o bem-estar humano, condicionando a sobrevivência das comunidades locadas em sua circunvizinhança, principalmente. É nesse sentido, que se evidencia a necessidade da promoção 
do uso racional e sustentável desse ambiente, visando a conservação do mesmo para o usufruto dos serviços ecossistêmicos prestados pelo ecossistema por parte das comunidades locais atuais e grupos sociais futuros. Portanto, deve-se reconhecer tais serviços e conscientizar a sociedade sobre sua importância nos âmbitos ambiental e social, a conscientização buscar alternativas de mitigação e/ou compensação dos impactos negativos decorrentes dos mais variados tipos de uso e ocupação desses espaços.

\section{REFERÊNCIAS}

ALONGI, D. M. Present state and future of the world's mangrove forests. Environmental Conservation, v. 29, n. 03, p. 331-349, 2002.

BANDARANAYAKE, W. M. Traditional and medicinal uses of mangroves. Mangroves and Salt Marshes, 2, 133-148, 1998.

BUNT, J. S. Overlap in mangrove species zonal patterns: some methods of analysis. Mangroves and Salt Marshes, v. 3, p. 155-164, 1999.

COSTA, D. F. S.; ROCHA, R. M.; CESTARO, L. A. Análise fitoecológica e zonação de manguezal em hipersalino. Mercator, v. 13, n. 1, p. 119-126, 2014.

COSTA, O. R. Zoneamento Ecológico-econômico da bacia hidrográfica do Ceará-Mirim/RN. 2005. Dissertação (Mestrado). Programa de Pós-Graduação em Geografia, Universidade Federal do Rio Grande do Norte, 2005.

DAWES, C. Mangrove structure, litter and macroalgal productivity in a northern-most forest of Florida. Mangroves and Salt Marshes, v. 03, n. 04, p. 259-267, 1999.

DEBENAY, J. P.; PAGÈS, J.; DIOUL, P. S. Ecological zonation of the hypersaline estuary of the Casamance River (Senegal): Foraminifera, zooplankton and abiotic variables. Hydrobiologia. v. 174, p. 161-176, 1989

DIAS, T. L. P. Os peixes, a pesca e os pescadores a Reserva de Desenvolvimento Sustentável Ponta do Tubarão (Macau-Guamaré/RN), Brasil. Tese (Doutorado), Programa de Pós-Graduação em Ciências Biológicas (Zoologia), Universidade Federal da Paraíba. João Pessoa, 2006.

DIAS, T. L. P.; ROSA, R. S.; DAMASCENO, L. C. P. Aspectos socioeconômicos, percepção ambiental e perspectivas das mulheres marisqueiras da Reserva de Desenvolvimento Sustentável Ponta do Tubarão (Rio Grande do Norte, Brasil). Gaia Scientia, v. 1, n. 1, p. 25-35, 2007.

DUKE, N. C, et al. A world without mangroves? Science, v. 317, n. 5834, p. 41-42, 2007. 
FALCÃO, M. G. A ictiofauna em planícies de maré nas baías das Laranjeiras e de Paranaguá, Paraná. Dissertação (Mestrado). Programa de Pós-Graduação em Ciências Biológicas-Área Zoologia, Universidade Federal do Paraná, 2005.

HAINES-YOUNG, R. H.; POTSCHIN, M.B. Ecosystem services: Exploring a geographical perspective. Progress in Physical Geography, v. 35, n. 5, p. 575-594, 2011.

HAINES-YOUNG, R.; POTSCHIN, M. Common International Classification of Ecosystem Services (CICES): Consultation on Version 4, August-December. EEA - Framework Contract, n. EEA/IEA/09/003. Nottingham: University of Nottingham/Centre for Environmental Management, 2013.

INSTITUTO DE DESENVOLVIMENTO SUSTENTÁVEL E MEIO AMBIENTE DO RIO GRANDE DO NORTE - IDEMA. Relatório Técnico para o Plano de Manejo da Reserva de Desenvolvimento Sustentável Estadual Ponta do Tubarão. Documento interno, 2008.

LIMA, P. G. Uma prática de educação ambiental para alunos do $3^{\circ}$ grau no manguezal do rio CearáMirim/Extremoz. Dissertação (Mestrado). Programa Pós-Graduação e Pesquisa em Geografia. Universidade Federal do Rio Grande do Norte, Natal, 2005.

LUGO, A. E. Mangrove ecosystems: successional or steady state. Biotropica, v. 12, n. 02, p. 65-72, 1980.

MATTOS, P. P.; KONIG, A.; FREIRE, F.A.M.; ALOUFA, M.A.I. Etnoconhecimento e percepção dos povos pesqueiros da Reserva Ponta do Tubarão acerca do ecossistema manguezal. Revista Brasileira de Biociências, n. 10, p. 481-489, 2012.

MIRANDA, L. B.; CASTRO, B. M.; KJERFVE, B. Princípios de oceanografia física de estuários. São Paulo: Editora da Universidade de São Paulo, 2002. 210 p.

POLIDORO, B. A. The loss of species: mangrove extinction risk and geographic areas of global concern. PlosOne. v. 5, n. 4, p. 1-10, 2010.

QUEIROZ, R.N.M.; DIAS, T.L.P. Molluscs associated with the macroalgae of the genus Gracilaria (Rhodophyta): importance of algal fronds as microhabitat in a hypersaline mangrove in Northeastern. Brazil. Braz. J. Biol., v. 74, n. 3, p. 52-63, 2014.

RABELO, M. S. A cegueira do óbvio: a importância dos serviços ecossistêmicos na mensuração do Bem-Estar. Tese (Doutorado). Programa de Pós-graduação em Desenvolvimento e Meio Ambiente, Universidade Federal do Ceará, 2014.

ROCHA JÚNIOR, J. M. Avaliação ecológica-econômica do manguezal de Macau/RN e a importância da aplicação de práticas preservacionistas pela indústria petrolífera local. Dissertação 
(Mestrado). Programa de Pós-graduação em Ciência e Engenharia de Petróleo, Universidade Federal do Rio Grande do Norte, 2011.

SAVENIJE, H. H. G.; PAGÈS, J. Hypersalinity: a dramatic change in the hydrology of Sahelian estuaries. Journal of Hydrology, v. 135, p. 157-174. 1992.

SILVA, B. B.; LOPES, G. M.; AZEVEDO, P. V. Balanço de radiação em áreas irrigadas utilizando imagens Landsat 5 - TM. Revista Brasileira de Meteorologia, v. 20, n. 2, p. 243-252, 2005.

SIMIER, M.; BLANC. L.; ALIAUME, C.; DIOUF, P.S.; ALBARET, J. J. Spatial and temporal structure of fish assemblages in an "inverse estuary", the Sine Saloum system (Senegal). Estuarine Coastal and Shelf Science, v. 59, p. 69-86, 2004.

SOUZA, L. P.; SOUZA, A. I.; ALVES, F.; LILLEB $\varnothing$, A. I. Ecosystem services provided by a complex coastal region: challenges of classification and mapping. Scientific Reports, v. 6, n. 22782, 2016.

TOMLINSON, P. B. The botany of mangroves. Cambridge: Cambridge University Press, 1986. $413 \mathrm{p}$.

VANNUCCI, M. Os manguezais e nós: uma síntese de percepções. São Paulo: EDUSP, 2002. 244 p.

YE, Y.; TAM, N. F-Y.; LU, C-Y.; WONG, Y-S. Effects of salinity on germination, seedling growth and physiology of three salt-secreting mangrove species. Aquatic Botany, v. 83, 193-205. 2005.

\section{AGRADECIMENTOS}

A equipe agradece a CAPES pela concessão da bolsa de mestrado/PPGE-UFRN, assim como ao CNPq (MCTI/CNPQ/Universal Proc.447227/2014-9) e ao TRÓPIKOS - Grupo de Pesquisa em Geoecologia e Biogeografia de Ambientes Tropicais (UFRN), pelo auxílio financeiro e apoio nos trabalhos de campo e de gabinete.

Recebido em: 15/08/2018

Aceite para publicação em: 05/11/2018 